POS $\quad$ PROCEEDINGS

\title{
Precision electroweak physics at the $\mathrm{LHeC}$ and FCC-eh
}

\author{
Daniel Britzger* \\ Physikalisches Institut, Universiät Heidelberg, Germany \\ E-mail: britzger@physi.uni-heidelberg.de

\section{Max Klein} \\ Physics Department, University of Liverpool, UK \\ E-mail: mkleinahep.ph.liv.ac.uk
}

\begin{abstract}
Electroweak (EW) physics at the future electron-proton colliders LHeC and FCC-eh is studied. Simulated neutral-current and charged-current deep-inelastic scattering cross sections are employed for simultaneous determinations of the parton distribution functions of the proton together with the fundamental parameters of the EW theory, including their statistical and systematic uncertainties. Uncertainties of the $W$ and $Z$ boson masses are determined and compared to uncertainties obtained from HERA combined data. The LHeC data will allow for a determination of $m_{W}$ with an uncertainty of $17 \mathrm{MeV}$, and the FCC-eh with $10 \mathrm{MeV}$, thus exceeding the precision of the currently most precise single measurements. The LHeC or FCC-eh data will allow for a precision determination of the vector and axial-vector couplings of the light quarks to the $Z$-boson, with uncertainties being smaller by an order order of magnitude than current measurements. It is shown, that the measurements of the EW parameters are not limited by the precision of the parton distribution functions, which have also to be determined from the same data. The measurements of the inclusive DIS cross sections as a function of the four-momentum transfer squared $Q^{2}$ will allow for high precision tests of the scale dependence of the EW theory in the range from a few $\mathrm{GeV}$ up to the $\mathrm{TeV}$ regime from a single process.
\end{abstract}

XXV International Workshop on Deep Inelastic Scattering and Related Topics 3-7 April, 2017

University of Birmingham, $U K$

\footnotetext{
* Speaker.
} 


\section{Introduction}

Electron-proton collisions have been extensively studied by the H1 and ZEUS experiments at the HERA collider at center-of-mass energies of $\sqrt{s}=318 \mathrm{GeV}$. The LHeC [U] and FCC-eh are future proposed electron-proton colliders at CERN, with $\sqrt{s}$ of $1.3 \mathrm{TeV}$ or $3.5 \mathrm{TeV}$, respectively, thus exceeding significantly the previously studied kinematic region. Both facilities forsee the use of a longitudinally polarised electron beam with energy $E_{e}=60 \mathrm{GeV}$, which is collided with either protons from the LHC $\left(E_{p}=7 \mathrm{TeV}\right)$ or the FCC-pp $\left(E_{p}=50 \mathrm{TeV}\right)$. The large $\sqrt{s}$ and hugely increased integrated luminosities of up to $\mathrm{ab}^{-1}$ as compared to HERA will allow for precise measurements of the inclusive neutral-current and charged-current deep-inelastic scattering cross sections (NC and CC DIS) at virtualities $Q^{2} \gtrsim m_{W / Z}^{2}$ where electroweak (EW) effects are important. This article presents studies of EW effects in inclusive NC and CC DIS at the LHeC and FCC-eh. The sensitivity of inclusive DIS cross sections on fundamental EW parameters, such as the $W$ and $Z$-boson masses $\left(m_{W}\right.$ and $\left.m_{Z}\right)$ or the weak neutral couplings of the light quarks $\left(v_{u, d}\right.$ and $\left.a_{u, d}\right)$, are studied.

\section{Electroweak effects in NC and CC DIS}

NC interactions in the process $e^{ \pm} p \rightarrow e^{ \pm} X$ are mediated by virtual photons $(\gamma)$ or $Z$ bosons in the $t$-channel. CC interactions, having a neutrino in the final state, are mediated by $W$-bosons. The predictions for inclusive NC and CC DIS cross sections are expressed in terms of generalised structure functions. For NC DIS they are $\tilde{F}_{2}^{ \pm}, x \tilde{F}_{3}^{ \pm}$and $\tilde{F}_{\mathrm{L}}^{ \pm}$and the cross section is determined as

$$
\frac{d^{2} \sigma^{\mathrm{NC}}\left(e^{ \pm} p\right)}{d x d Q^{2}}=\frac{2 \pi \alpha^{2}}{x Q^{2}}\left[Y_{+} \tilde{F}_{2}^{ \pm}\left(x, Q^{2}\right) \mp Y_{-} x \tilde{F}_{3}^{ \pm}\left(x, Q^{2}\right)-y^{2} \tilde{F}_{\mathrm{L}}^{ \pm}\left(x, Q^{2}\right)\right]
$$

with $\alpha$ being the fine structure constant. The helicity dependence of the interactions is contained in the terms $Y_{\mp}=1 \pm(1-y)^{2}$ with $y$ being the inelasticity of the process, and $x$ is defined as $x=Q^{2} /(y s)$. The contributions from $F_{L}$ are only sizeable at high $y$ and thus not discussed in the following. The generalised structure functions are further decomposed into contributions from pure photon-exchange, pure $Z$-exchange and $\gamma Z$-interference and read

$$
\begin{aligned}
\tilde{F}_{2}^{ \pm} & =F_{2}-\left(v_{e} \pm P_{e} a_{e}\right) \kappa_{Z} F_{2}^{\gamma Z}+\left[\left(v_{e}^{2}+a_{e}^{2}\right) \pm 2 P_{e} a_{e}\right] \kappa_{Z}^{2} F_{2}^{Z} \\
x \tilde{F}_{3}^{ \pm} & =-\left(a_{e} \pm P_{e} v_{e}\right) \kappa_{Z} F_{3}^{\gamma Z}+\left[2 v_{e} a_{e} \pm P_{e}\left(v_{e}^{2}+a_{e}^{2}\right)\right] \kappa_{Z}^{2} F_{3}^{Z},
\end{aligned}
$$

with $P_{e}$ denoting the longitudinal polarisation of the electron beam and using for the normalisation $\kappa_{Z}$ and the Fermi coupling constant $\left(G_{\mathrm{F}}\right)$

$$
\kappa_{Z}\left(Q^{2}\right)=\frac{Q^{2}}{Q^{2}+m_{Z}^{2}} \frac{G_{\mathrm{F}} m_{Z}^{2}}{2 \sqrt{2} \pi \alpha} \text { and } G_{\mathrm{F}}=\frac{2 \pi \alpha}{2 \sqrt{2} m_{W}^{2}}\left(1-\frac{m_{W}^{2}}{m_{Z}^{2}}\right)^{-1}(1+\Delta r) .
$$

The term $\Delta r=\Delta r\left(\alpha, m_{W}, m_{Z}, m_{t}, m_{H}, \ldots\right)$ contains corrections to the muon decay and its calculation takes $\alpha$ and the boson and fermion masses as input [2]. The structure functions are related to linear combinations of the quark and anti-quark momentum distributions, $x q$ and $x \bar{q}$, and read in 
the quark-parton model

$$
\begin{aligned}
{\left[F_{2}, F_{2}^{\gamma Z}, F_{2}^{Z}\right] } & =x \sum_{q}\left[e_{q}^{2}, 2 e_{q} v_{q}, v_{q}^{2}+a_{q}^{2}\right]\{q+\bar{q}\}, \\
{\left[F_{3}^{\gamma Z}, F_{3}^{Z}\right] } & =x \sum_{q}\left[2 e_{q} a_{q}, 2 v_{q} a_{q}\right]\{q+\bar{q}\},
\end{aligned}
$$

where $a_{q}$ and $v_{q}$ are the weak neutral vector and axial-vector couplings of the quarks to the $Z$-boson. The couplings are predicted by the Standard Model (SM) to be $a_{q}=I_{q, L}^{(3)}$ and $v_{q}=I_{q, L}^{(3)}-2 e_{q} \sin ^{2} \theta_{W}$, with $I_{q, L}^{(3)}$ being the third component of the left-handed isospin. In the present study these couplings may be considered as additional parameters and thus have to be determined from the simulated, experimental data.

\section{Simulated NC and CC DIS data}

Inclusive NC and CC DIS data are simulated for the expected LHeC and FCC-eh running conditions. The simulated data sets comprise electron and positron beams with different energies and polarisation states, and different proton beam energies. A summary of the $\mathrm{NC}$ and $\mathrm{CC}$ data and the expected integrated luminosities is provided in table $\mathrm{W}$. A high polarisation will be of particular im-

\begin{tabular}{lccccc}
\hline lepton type & $E_{e}[\mathrm{GeV}]$ & $P_{e}$ & $E_{p}[\mathrm{TeV}]$ & $\mathcal{L}\left[\mathrm{fb}^{-1}\right]$ & Cross sections \\
\hline$e^{-}$ & $60(60)$ & -0.8 & $50(7)$ & 1000 & NC, CC \\
$e^{-}$ & $60(60)$ & +0.8 & $50(7)$ & 300 & NC, CC \\
$e^{+}$ & $60(60)$ & 0 & $50(7)$ & 100 & NC, CC \\
$e^{-}$ & $20(60)$ & 0 & $7(1)$ & 100 & NC, CC \\
\hline
\end{tabular}

Table 1: Simulated data sets for different beam parameters and their expected luminosities for FCC-eh and $\mathrm{LHeC}$ (in parenthesis).

portance for a precise determination of the weak neutral vector couplings of the light quarks. The assumptions made for the individual sources of the uncertainties are summarised in table $\nabla$. The

\begin{tabular}{lc}
\hline Source of uncertainty & Error on source or cross section \\
\hline Scattered electron energy scale $\Delta E_{e}^{\prime} / E_{e}^{\prime}$ & $0.1 \%$ \\
Scattered electron polar angle & $0.1 \mathrm{mrad}$ \\
Hadronic energy scale $\Delta E_{h} / E_{h}$ & $0.5 \%$ \\
Calorimeter noise (only $y<0.01)$ & $1-3 \%$ \\
Radiative corrections & $0.3 \%$ \\
Photoproduction background (only $y>0.5)$ & $1 \%$ \\
Global efficiency uncertainty & $0.5 \%$ \\
\hline
\end{tabular}

Table 2: Assumptions imposed on the size of uncertainties for the simulated NC DIS cross sections.

size of uncertainties is typical inline with the best values achieved by H1 at HERA, and thus may be considered to be conservative as advanced detector concepts and hugely increased luminosities should yield significantly reduced uncertainties. 

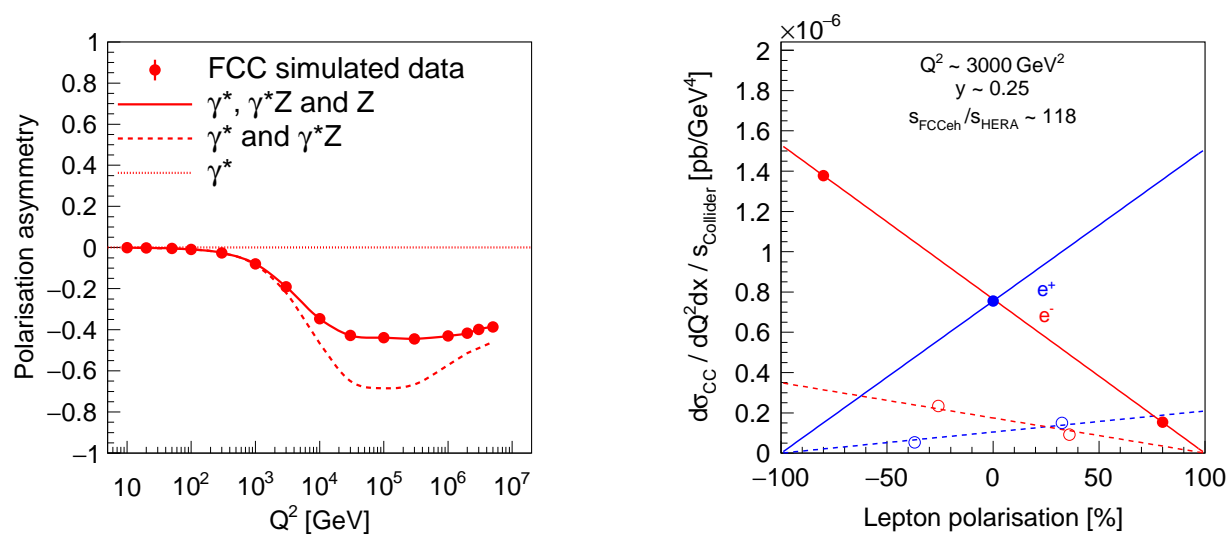

Figure 1: Left: Neutral-current polarisation asymmetry as a function of $Q^{2}$ integrated over $x$ for FCCeh simulated data. The polarisation asymmetry is displayed for pure photon exchange, which is zero by definition, for calculations including only the interference terms $\gamma^{*} Z$, and for predictions including also purely weak effects, $Z Z$. The full circles illustrate simulated data points, which have uncertainties invisible at the chose scales. Right: Charged-current cross sections measured with different lepton polarisation states and for electron (red) and positron (blue) beams. The full circles illustrate FCC-eh simulated data, whereas the open circles show H1 measurements. The data are scaled by the the center-of-mass energies of the respective collider. The error bars are smaller than the markers.

The size of electroweak contibutions to the NC DIS cross sections may be expressed by the polarisation asymmetry, defined as

$$
A=\frac{2}{P_{L}-P_{R}} \frac{\sigma\left(P_{L}\right)-\sigma\left(P_{R}\right)}{\sigma\left(P_{L}\right)+\sigma\left(P_{R}\right)},
$$

using the cross sections measured for two distinct beam polarisations $P_{L}$ and $P_{R}$. Its dependence as a function of $Q^{2}$ for simulated FCC-eh data is displayed in figure $\mathbb{W}$ (left), where calculations involving exclusively $\gamma$-exchange, or $\gamma$ and $\gamma Z$-exchange are displayed in addition. The future FCC-eh data will grossly extent measurements by HERA, which did not exceed $Q^{2} \gtrsim 10^{4} \mathrm{GeV}^{2}$, to the regions where contributions from purely $Z$-exchange are relevant.

The simulated CC DIS cross sections for the different beam conditions at a given value of $Q^{2}$ and $y$ are compared to data by $\mathrm{H} 1$ in figure $\mathrm{W}$ (right). At the displayed value of $Q^{2}=3000 \mathrm{GeV}^{2}$, the FCC-eh cross sections will be much higher than at HERA. At the selected $Q^{2}$ value, helicity effects of CC cross sections are irrelevant for FCC-eh and thus $e^{+}$and $e^{-}$cross sections are of the same size for unpolarised beams because they are at low- $x$ and thus mainly gluon initiated.

\section{Determination of EW parameters}

\subsection{Methodology of a combined EW and QCD fit}

The simulated NC and CC DIS data are employed for a determination of the parameters of the EW theory. EW parameters are determined in a simultaneous fit together with parameters of the parton distribution functions of the proton (PDFs), denoted as 'PDF+EW-fit'. This is because the 

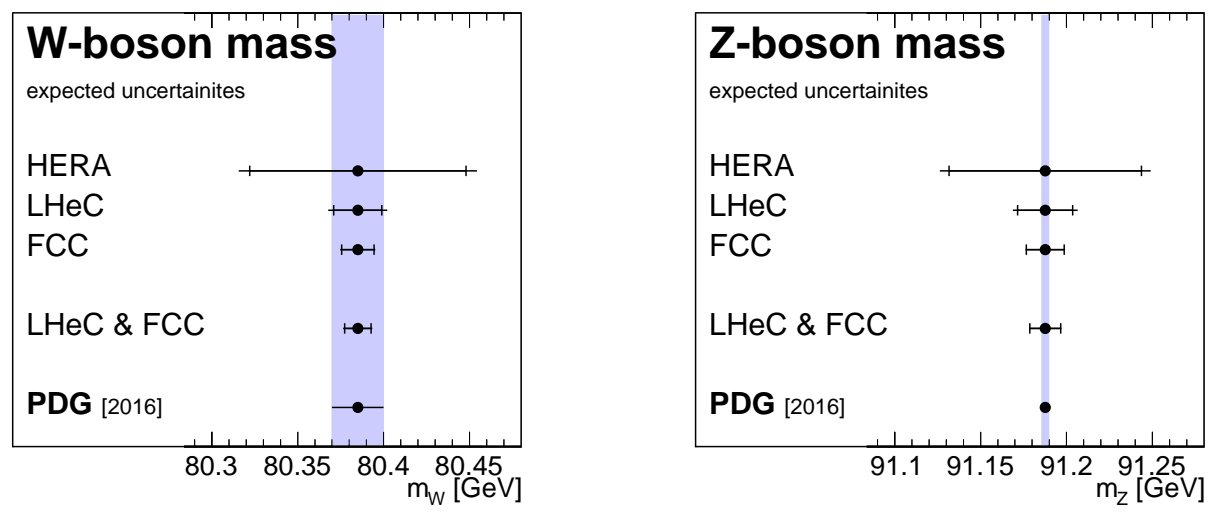

Figure 2: Measurements of the $W$-boson mass (left) and $Z$-boson mass (right) from HERA, LHeC and FCC-eh (simulated) data and compared to the PDG values.

PDFs have to be determined from the same data, and by performing a simultaneously determination of the PDFs and EW parameters the uncertainties of the PDFs are accounted for accordingly. The fitting methodology follows closely previous approaches [ [], [, [1] and it is observed that the prospects for the EW parameters are insensitive to details of the PDF fit methodology. The calculations are performed in the on-shell scheme, where the theory is expressed in terms of $\alpha, m_{W}, m_{Z}$, and $\Delta r$.

\subsection{W-boson, Z-boson and top-quark masses}

The uncertainty values of $m_{W}$ and $m_{Z}$ are determined in the PDF+EW-fit, where one of the masses is determined together with the PDFs, while the other boson mass is taken as external input. The expected uncertainties are displayed in figure $\square$ and compared to the PDG values [ 6 ], and to the uncertainties obtained when performing our PDF+EW-fit to the final combined HERA data [3]]. The expected uncertainties of $m_{W}$ are

$$
\begin{aligned}
\Delta m_{W}(\mathrm{LHeC}) & = \pm 14_{(\mathrm{exp})} \pm 10_{(\mathrm{PDF})} \mathrm{MeV} \text { and } \\
\Delta m_{W}(\mathrm{FCC}-\mathrm{eh}) & = \pm 9_{(\mathrm{exp})} \pm 4_{(\mathrm{PDF})} \mathrm{MeV},
\end{aligned}
$$

for LHeC and FCC-eh, respectively, where the breakdown into experimental and PDF uncertainties is obtained by repeating the fit with PDF parameters fixed. The expected uncertainties of $m_{Z}$ are about $19 \mathrm{MeV}$ and $11 \mathrm{MeV}$ for LHeC and FCC-eh, respectively, and are thus of similar size than those of $m_{W}$. The expected precision of $m_{Z}$ can not compete with the precise measurements at the $Z$-pole by the LEP and SLC experiments, but the future $e p$ facilities will test the SM much more precisely than hitherto, and they will improve significantly the current precision of $m_{W}$.

A simultaneous determination of $m_{W}$ and $m_{Z}$ together with the PDFs is performed and results are compared to a determination from $\mathrm{H} 1$ [1] in figure B] (left). Due to the large correlation between $m_{W}$ and $m_{Z}$, HERA data is not sufficient to determine those values reliably. Contrarily, the highly increased center-of-mass energy of LHeC or FCC-eh will allow for such a simultaneous determinaton of $m_{W}$ and $m_{Z}$ with high precision. 

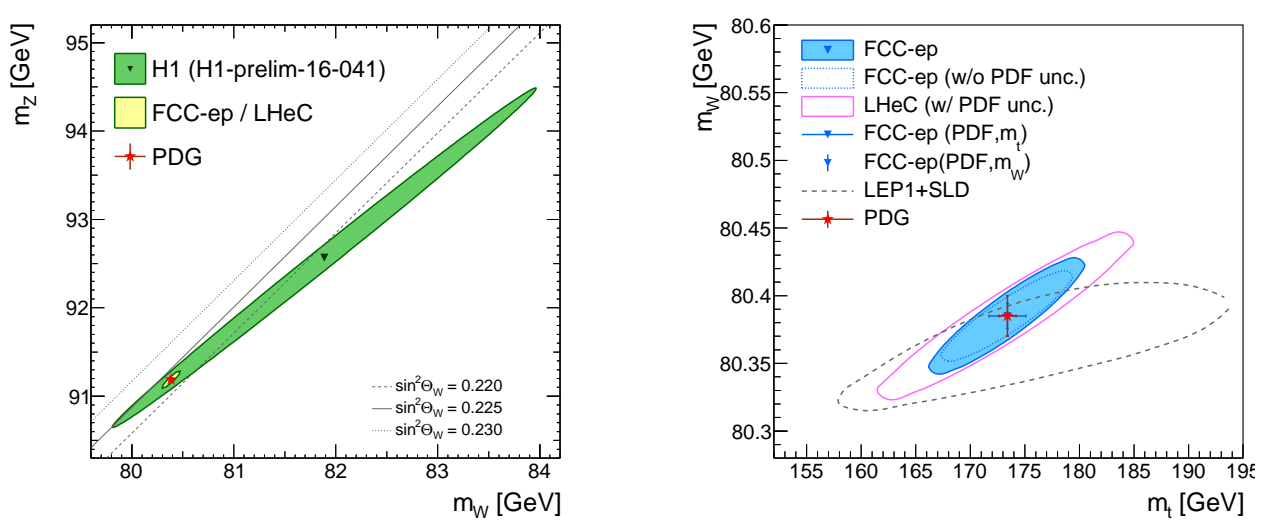

Figure 3: Simultaneous determination of $m_{W}$ and $m_{Z}$ from simulated LHeC and FCC-eh data in comparison to a determination by $\mathrm{H} 1$ (left). The thin lines illustrate the corresponding value of $\sin ^{2} \theta_{W}$ in the onshell scheme. Simultaneous determination of $m_{W}$ and $m_{t}$ from simulated LHeC and FCC-eh data (left) in comparison to the achieved precision by the LEP+SLD combination.

The top-quark mass $m_{t}$ contributes to the calculations through $\Delta r$. A simultaneous determination of $m_{W}$ and $m_{t}$, where $m_{Z}$ is an external input, is presented in figure B (right) and compared to results from LEP+SLD [ $[$ ]. Both, the LHeC and FCC-eh, will be able to improve the combined results from the LEP and SLC experiments, and thus provide a very sensitive test of the EW sector of the SM. The indirect constraints from inclusive DIS LHeC (FCC-eh) data will allow to determine $m_{t}$ with an uncertainty of $1.8 \mathrm{GeV}(1.5 \mathrm{GeV})$. Additional direct measurements of $m_{t}$ will significantly improve these results.

\subsection{Weak neutral couplings of light quarks}

The vector and axial-vector couplings of up-type and down-type quarks to the $Z$ ( $v_{u / d}$ and $a_{u / d}$ ) are determined in a single fit of the four couplings together with the PDFs. Resulting uncertainties are presented in table B] and compared to the currently most precise measurements [6]. The two-

\begin{tabular}{cccc}
\hline Coupling & PDG & \multicolumn{2}{c}{ Expected uncertainties } \\
parameter & & LHeC & FCC-eh \\
\hline$a_{u}$ & $0.50_{-0.05}^{+0.04}$ & 0.006 & 0.003 \\
$a_{d}$ & $-0.514_{-0.029}^{+0.050}$ & 0.011 & 0.005 \\
$v_{u}$ & $0.18_{ \pm 0.05}^{ \pm 0.003}$ & 0.003 \\
$v_{d}$ & $-0.35_{-0.06}^{+0.05}$ & 0.008 & 0.005 \\
\hline
\end{tabular}

Table 3: Standard model expectations for the light-quark weak neutral couplings $\left(a_{u}, a_{d}, v_{u}, v_{d}\right)$ together with the currently most precise uncertainties (PDG [目]) and the prospected uncertainties for LHeC and FCC-eh.

dimensional uncertainty contours $\left(\Delta \chi^{2}=2.3\right)$ are displayed in figure $⿴$ for each single quark flavor and compared to recent measurements. While the current determinations from $e^{+} e^{-}, e p$ or $p \bar{p}$ data have all somewhat similar precision, the future $e p$ facilities will greatly improve the precision of the weak neutral couplings and expected uncertainties are an order of magnitude smaller than the currently most precise ones [焑]. 

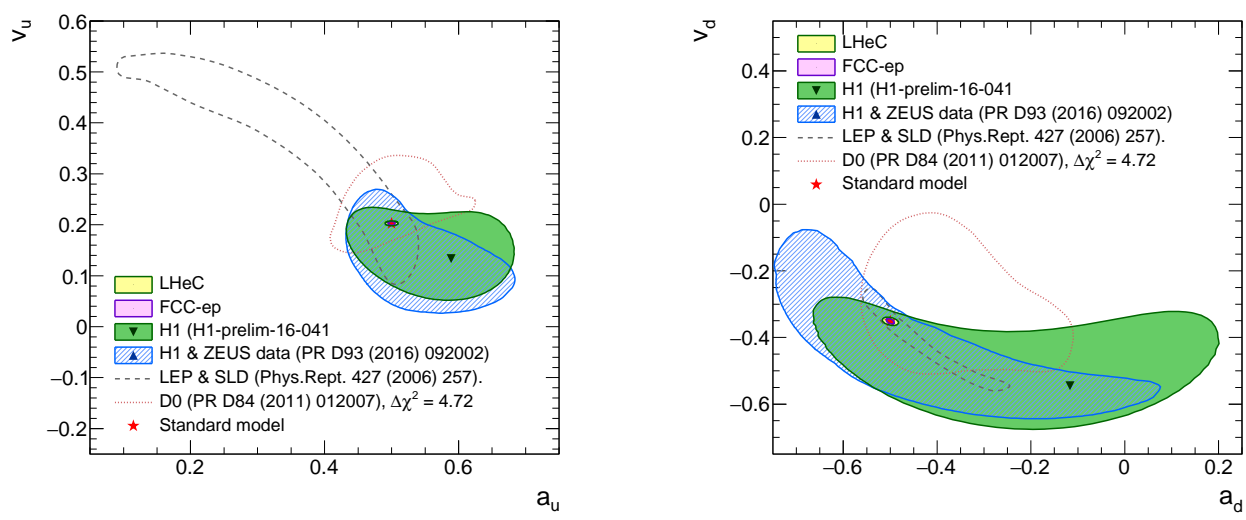

Figure 4: Results for the vector and axial-vector couplings of the up-type (left) and down-type (right) quarks to the $Z$ at the $68 \%$ confidence level (C.L.) for simulated LHeC and FCC-eh data. The results are compared to measurements by H1 [[5] and D0 [ [ ] ], and to determinations using H1 and ZEUS [Q] or LEP and SLD data [四]. The standard model expectations are diplayed by a red star.

\subsection{Scale dependence of $\sin ^{2} \theta_{W}$}

The inclusive DIS cross sections are measured over a large kinematic range. This allows for a unique test of the scale dependence of EW effects from a single process. A fit of PDF parameters together with 14 parameters representing the weak-mixing angle $\sin ^{2} \theta_{W}$ at individual $Q^{2}$ values of the simulated data is performed. The results are presented in figure [5. In the SM, no scale dependence is built in in the on-shell scheme since $\sin ^{2} \theta_{W}$ can be equivalently expressed as a determination of $m_{W}$. In this case the prospected precision would be around $10 \mathrm{MeV}(15 \mathrm{MeV})$ over a large kinematic range for the FCC-eh (LHeC).

This study, which is to be performed in the $\overline{\mathrm{MS}}$ scheme next, well illustrates the expected sensitivity to EW effects at the different scales in the range from $10 \mathrm{GeV}$ up to the $\mathrm{TeV}$ regime.

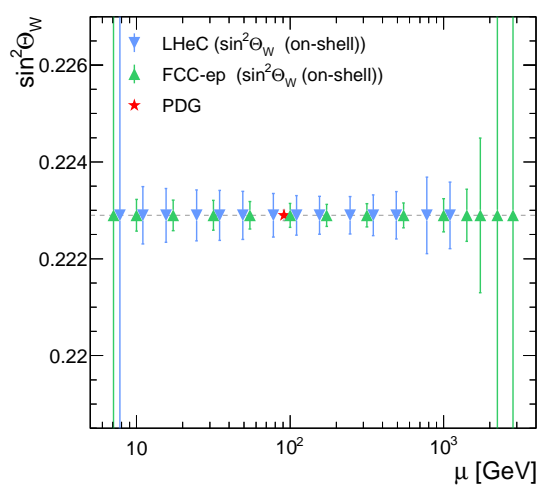

Figure 5: The weak-mixing angle $\sin ^{2} \theta_{W}$ in the on-shell scheme definition at different values of $Q^{2}$.

\section{Summary and conclusion}

Simulated neutral-current and charged-current DIS data are employed for a determination of the fundamental parameter of the electroweak theory. The LHeC or FCC-eh will allow for precision measurements of the electroweak sector, by far exceeding the precision of HERA measurements and in general with comparable or even higher precision than the LEP or LHC experiments. These measurements thus provide complementary tests of the Standard Model in the domain of spacelike momentum transfer. The measurements of the EW parameters are not limited by the precision 
of the parton distribution functions, which have also to be determined from the same data. It is demonstrated, that the $\mathrm{LHeC}$ or FCC-eh will allow for a very precise determination of the lightquark weak couplings. Whether with that new level of precision deviations from the SM coupling predictions may be revealed is for future $e p$ data to tell.

\section{References}

[1] LHeC Study group, J.L. Abelleira et al., J.Phys. G39 (2012) 075001, arXiv:1206.2913.

[2] H. Spiesberger, "EPRC. A program package for electroweak physics at HERA," in proceedings of the 95-96 Workshop on Future Physics at HERA, part 2. 1995.

[3] H1 and ZEUS Collaboration, H. Abramowicz et al., Eur. Phys. J. C75 (2015) 580, arXiv:1506.06042.

[4] M. Klein and V. Radescu, "Partons from the LHeC," CERN-LHeC-Note-2013-002, Jul 2013, https://cds.cern.ch/record/1564929.

[5] H1 Collaboration, H1-prelim-16-041, 2016, ava1lable online.

[6] Particle Data Group Collaboration, C. Patrignani et al., Chin. Phys. C40 (2016) 100001.

[7] ALEPH, DELPHI, L3, OPAL and SLD Collaborations, S. Schael et al., Phys. Rept. 427 (2006) 257, hep-ex/0509008.

[8] D0 Collaboration, V.M. Abazov et al., Phys.Rev. D84 (2011) 012007, arXiv: 104.4590.

[9] ZEUS Collaboration, H. Abramovicz et al., Phys.Rev. D93 (2016) 092002. 